\title{
The problem of quantization of lightcone QCD
}

\author{
Alexey Popov* \\ Novgorod SU (Velikiy Novgorod, Russia) \\ E-mail: avp@novgorod.net
}

This paper is devoted to the recent progress in the method of the lightcone coordinates in QCD. We show that boundary gauge fields are crucial for the consistent and complete definition of the theory. The result is important for the theory of high energy QCD evolution, since scattering amplitudes are directly related to the lightcone Hamiltonian, whose complete structure is still unclear on quantum level. Namely, there exists the problem to construct a quantum algebra of observables in lightcone QCD beyond the perturbative regime. Careful analysis shows that we have the problems with: canonical commutation relations, spatial invariance, and the boundary degrees of freedom in the Hamiltonian.

The XXth International Workshop High Energy Physics and Quantum Field Theory September 24 - October 1, 2011

Sochi, Russia

${ }^{*}$ Speaker. 


\section{Introduction}

There exists three main theoretical methods in high energy QCD: feynman diagrams (Lipatov, Braun, Kovchegov), path integral (McLerran, Hatta, Balitski), quantum Hamiltonian (Kovner, Lublinsky, Mueller). There are two typical coordinates in the Hamiltonian method: cartesian coordinates, lightcone coordinates. In this work we study the method of lightcone coordinates The main advantages of the lightcone method are: only operator algebra, no diagrams, life in the physical Hilbert space directly related to observables, trivial vacuum, correctly removed gauge freedom, no ghosts, no zero modes. Briefly, the primary physical motivation of our studied is: the evolution of amplitudes of high energy scattering in QCD, JIMWLK/BK equations and NLO corrections, powerful generalization of BFKL in the framework of evolution equations, unification of pomeron loops and pomeron poles, multiple gluon production and other exclusive processes, a possible way to the nonperturbative area of QCD.

However, a further progress is difficult due to subtle mathematical problems in the formalism, which should be solved. It was shown recently that in lightcone QCD the boundary gauge fields at $x^{-}= \pm \infty$ are important part of the theory $[1,2,3]$. Since there is no natural boundary condition in QCD, we must carefully treat boundary fields in the construction of the quantum theory. The practical importance of the $x^{-}$-boundaries was established in Ref. [2] by the considering of the theory of high energy evolution. However, the theoretical input used in Ref. [2] is not fully verified by a rigorous formalism. The construction of a such formalism had been started in Ref. [1], where the classical lightcone QCD is considered, but the problem of quantization was not examined in detail. Hoping to stimulate future researches, in this paper we analyze the currently known attempts to quantize the theory and show that all they fail.

Refs. [3, 4] are the first known papers where the role of a field asymptotic was established. However, in those papers only the antisymmetric boundary condition was used. Nowadays, we know [1] that in QCD the boundary condition is neither antisymmetric nor symmetric. In Ref. [2] the boundary fields are used in the theory of high energy scattering, since the method of wave function requires the precisely defined quantum Hamiltonian. The complete description of the lightcone QCD at the classical level was given in Ref. [1]. Nevertheless, a quantization procedure of the obtained system is not specified yet. The standard way of quantization is a construction of a Poisson algebra. The key problem here is that a Poisson algebra of bulk fields is unable to treat boundary fields. Finally, a Poisson bracket should be quantized and a representation of the quantized algebra in a Hilbert space should be found. For a theory without boundary fields this procedure is simple enough. However, even for a slightly more complex theory, the procedure may become unsolvable. In this paper we show that in lightcone QCD a Poisson algebra cannot be directly constructed, since the Hamiltonian has both the explicit and the implicit boundary contributions. Hence, we have to find a workaround that can help to construct the algebra. We analyze all three known choices of variables, which were used to construct the required Poisson algebra. The result is negative, the standard method of quantization cannot be applied.

This paper is organized as follows. In Sec. 2, we briefly review lightcone QCD at the classical level. In Sec. 3, we analyze the available choices of coordinates with aim to construct a Poisson algebra and to quantize it. Section 4 contains our conclusions. 


\section{Lightcone QCD at the classical level}

Let us briefly review the results of Ref. [1]. The Lagrangian of QCD is

$$
L=\frac{1}{2} F_{+-}^{2}+F_{+i} F_{-i}-\frac{1}{4} F_{i j} F^{i j}+g A_{\mu} J^{\mu}
$$

where $J^{\mu}$ is a current of matter fields or an external current. To remove unphysical degrees of freedom we apply the symplectic Faddeev-Jackiw method [6]. This method allows to do the job without a construction of a Poisson algebra, which has a problem with boundary degrees of freedom.

In the process of symplectic reduction, since there is no term $\partial_{+} A_{+}$in the Lagrangian, the variables $A_{+}$are automatically removed from the scene. The main gauge fixing is

$$
A_{-}=0
$$

The necessity to reduce the Hamiltonian on the phase space without $A_{+}$gives the complete set of Gauss' constraints.

$$
\begin{gathered}
\partial_{-} \pi_{a}^{-}+\partial_{-} \partial_{i} A_{i}^{a}-g f_{a b c} \partial_{-} A_{i}^{b} A_{i}^{c}+g J_{a}^{+}=0 \\
\pi_{a}^{-}( \pm \infty, \vec{x})=0
\end{gathered}
$$

where the last constraint comes from the boundary part of the variation $\delta H$ over $\delta A_{+}$.

After the removal of unphysical degrees of freedom, we obtain the following classical dynamical system. The phase space of the theory is the space of fields $A_{i}^{a}\left(x^{-}, \vec{x}\right)$ obeying the boundary condition

$$
A_{i}^{a}(-\infty, \vec{x})=0
$$

This condition was selected in Ref. [1] as a most simple way to fix the residual gauge freedom. The linear space of fields $A_{i}^{a}$ is endowed by the fundamental symplectic form $\omega$

$$
\begin{gathered}
\omega=\int \partial_{-} d A_{i}^{a} \wedge d A_{i}^{a} \\
\omega(A, B)=\int\left(\partial_{-} A_{i}^{a} B_{i}^{a}-A_{i}^{a} \partial_{-} B_{i}^{a}\right) d x^{-}
\end{gathered}
$$

The Hamiltonian density is given by

$$
H\left[A_{i}\right]=\frac{1}{2}\left(\pi_{a}^{-}\right)^{2}+\frac{1}{4} F_{i j}^{a}\left[A_{i}\right] F_{a}^{i j}\left[A_{i}\right]-g A_{i}^{a} J_{a}^{i}
$$

The momentum $\pi_{a}^{-}\left(x^{-}, \vec{x}\right)$ is determined by the equations (2.3) and (2.4), which is the Gauss' constraints. At the boundary $x^{-}=+\infty$ we have the constraint

$$
\begin{gathered}
A_{i}^{a}(+\infty, \vec{x})=\gamma_{i}^{a}(\vec{x}) \\
\partial_{i} \gamma_{i}^{a}(\vec{x})=\int_{-\infty}^{+\infty}\left(g f_{a b c} \partial_{-} A_{i}^{b} A_{i}^{c}-g J_{a}^{+}\right) d x^{-}
\end{gathered}
$$


which is the consequence of the Gauss' constraints (2.3) and (2.4). We also add the following constraint

$$
\partial_{i} \gamma_{j}^{a}-\partial_{j} \gamma_{i}^{a}+g f_{a b c} \gamma_{i}^{b} \gamma_{j}^{c}=0
$$

which is the finite energy condition for infinity length of $x^{-}$. This condition is introduced manually to complete fix $\gamma_{i}^{a}$. Formally, the constraint (2.11) does not arise in the symplectic Faddeev-Jackiw algorithm. So, it must be used carefully, since in a quantum theory energy levels have a sense only relatively to a ground state. If we remove the constraint (2.11), then it will be possible to replace

$$
\gamma_{i}^{a} \rightarrow \gamma_{i}^{a}+\varepsilon^{i j} \partial_{j} \psi^{a}
$$

where $\psi^{a}(\vec{x})$ is an arbitrary function. Non zero value of $\partial_{i} \psi^{a}$ leads to infinity (volume-divergent) energy in the Hamiltonian (2.8). Note, that the transformation (2.12) does not violate the constraint (2.10). So, in this case the variable $\psi$ must be considered as a boundary degree of freedom.

\section{The problem of quantization}

Initially, we have a symplectic system. Note, that in the classical mechanic the symplectic form $d p \wedge d q$ is a natural part of the construction of the Hamiltonian formalism. Next, the Poisson bracket $\{q, p\}=1$ is obtained via the implicit inversion on the symplectic form. Usually, this step is not emphasized because it is trivial. However, subtleties may emerge in an infinite-dimensional field theory and in a constrained system.

In a quantum theory, a role of Poisson algebra is much more important because a quantum commutator is a quantized version of the classical brackets. The mainstream method of quantization is

1. To construct a Poisson algebra and a Hamiltonian. This algebra will be used as an algebra of classical observables.

2. To fix a gauge invariance and to remove constraints via a construction of the Dirac bracket.

3. To quantize the Poisson algebra. To construct a Hilbert space and canonical commutation relations.

4. To resolve possible ordering ambiguities in quantum-classical operator correspondence.

The second step we perform within symplectic system. To make the first step we analyze the three variables with aim to generate well defined and quantizable Poisson algebra.

\subsection{Natural variables $A_{i}^{a}$}

To construct a Poisson algebra from our symplectic dynamical system we have to invert the symplectic form (2.7) and to construct a Poisson brackets. Let us try to invert the symplectic form in the linear space of fields obeying the boundary condition $A_{i}^{a}(-\infty, \vec{x})=0$. We have to find a linear operator $P(x-y)$ such that

$$
\omega\left(A, \int P(x-y) B(y) d y\right)=\int A B d x
$$


for any $A$ and $B$ obeying (2.5). Note that we have omit the color and transverse indexes. Then, the corresponding Poisson brackets is

$$
\{F, G\}=\int \frac{\delta F[A]}{\delta A(x)} P(x-y) \frac{\delta G[A]}{\delta A(y)} d x d y
$$

It is easy to check that for the boundary condition (2.5) there exists only one such operator

$$
P(x)=\frac{1}{2} \theta(-x)
$$

where $\theta(x)$ is the standard step function. The result (3.3) has a fatal problem: the kernel $P(x)$ is not antisymmetric. This obstruction is expected specific feature of a infinite-dimensional theory with boundaries. Hence, a naive Poisson brackets does not exist and the variables $A_{i}^{a}$ cannot generate a Poisson algebra of observables. In principle, this way is not fully closed and it may be exist a subtle method of a construction of algebra.

\subsection{Antisymmetric variables $\tilde{A}_{i}^{a}$}

From the wide practice of lightcone field theories we know that the antisymmetric conditions induces a well-defined Poisson algebra. The symplectic form $\omega$ is invertible and the corresponding inverse kernel is

$$
P(x)=-\frac{1}{4} \varepsilon(x)
$$

where $\varepsilon(x)$ is the sign function. However, the fatal problem here is that the lightcone QCD Hamiltonian has an implicit dependence over the boundary field $\gamma_{i}$. To extract it, we calculate an infinitesimal variation $\delta \tilde{H}$ over a variation of the canonical variables $\delta \tilde{A}$. It is shown in Ref. [10] that the boundary contribution to the variation $\delta \tilde{H}$ is

$$
\left.\delta \tilde{H}\right|_{\text {boundary }}=-\int_{x^{-}, \vec{x}} \frac{g}{4} f_{a b c} \pi_{a}^{-} \delta \gamma_{i}^{b} \gamma_{i}^{c} \sim O\left(g^{3}\right)
$$

The boundary variation cannot be converted to a bulk one, since there exists an obstruction that the color-space 1-form $f_{a b c} d \gamma^{b} \gamma^{c}$ is not exact and not closed, except the case of a one-dimensional color space. So, the Hamiltonian is not an element of the bulk Poisson algebra and the standard method of quantization can not be applied.

\subsection{Boundaryless variables $c_{i}^{a}$}

The another attempt to separate the boundary contribution is proposed in Ref. [2]. The idea is to express a boundary contribution to a bulk one. Let us define new fundamental variables $c_{i}^{a}$ with zero boundary conditions as

$$
A_{i}^{a}\left(x^{-}, \vec{x}\right)=c_{i}^{a}\left(x^{-}, \vec{x}\right)+\gamma_{i}^{a}(\vec{x}) \varphi\left(x^{-}\right)
$$

where $\varphi\left(x^{-}\right)$is an arbitrary fixed global function such that

$$
\begin{aligned}
& \varphi(-\infty)=0 \\
& \varphi(+\infty)=1
\end{aligned}
$$


It is helpful to imagine $\varphi\left(x^{-}\right)$as a typical smooth monotonic kink-like function. So, we have $c_{i}^{a}( \pm \infty)=0$. This gives the new Hamiltonian $H[c]$, which variation $\delta H[c]$ is well defined [10].

The first problem of this method is that the fundamental brackets $\left\{c\left(x_{1}\right), c\left(x_{2}\right)\right\}$ becomes very nonlinear. Although we have no methods how to quantize this nonlinear brackets, in principle, we can hope that a solution exist. The usage of variables $c_{i}^{a}$ has the one more problem. Since the function $\varphi\left(x^{-}\right)$explicitly depends on $x^{-}$, it breaks the longitudinal spatial invariance of the Hamiltonian. This problem is most serious because there are no preferred points in the initial formulation of the theory.

\section{Discussion}

We have analyzed the three possible variables: $A, \tilde{A}$, and $c$. All they give the fatal obstructions that do not allow to quantize the theory in the standard Poisson method. This motivates us to find a different quantization scheme that is not based on a bulk Poisson algebra. In Refs. [7, 8] it was proposed a generalization of Poisson brackets to a case with boundaries. It is possible to construct a consistent Poisson bracket that includes the full boundary information. For boundaryless functionals a such bracket coincides with the standard Poisson brackets, but for functionals, involving boundaries, the brackets are defined in a distinct way. Although this generalized bracket seem challenging, there are no hopes to quantize the such brackets in a trivial way. Moreover, it was shown that this brackets are not unique $[8,9]$. It is not clear what brackets naturally corresponds to a symplectic structure obtained from a Lagrangian. The task becomes more complex if we impose the constraint $\tilde{A}( \pm \infty)= \pm \gamma / 2$ that must be treated as a second class constriant.

Another quantization method, which we did not touch before, is an introduction of unphysical degrees of freedom into the Hilbert space of the quantum theory. Sometimes, such methods leads to unitary ghosts and Faddeev-Popov ghosts in a Hilbert space. A selection of physical states is given by special operator conditions, whose matrix elements gives a system of linear equations. The key problem in this way is that to relate a such enlarged Hilbert space to the real physical states observed in high energy scattering. Even after an explicit removal of ghost states, a physical interpretation is still not clear.

Since we did not assume any perturbative approximation or expansion, our calculation is valid for any value of the coupling constant $g$. Indeed, we have shown that near $g \approx 0$ the theory can be quantized well. This means that nonperturbative aspects of lightcone QCD should arise somewhere. Recall that any lightcone theory has the trivial vacuum state. So, in a general case, potential troubles are moved to a very complicated Hamiltonian and to a quantization procedure. We just conjecture that the observed obstructions is not technical but a genuine feature of the lightcone theory. It is suitable to remind here about the lightcone quantization of the bosonic string theory where the critical dimension is given by a cumbersome check of the commutation relations of the Lorentz group. In lightcone QCD we also see the drastic growing of complexity when we increase the dimension of space-time from three to four. Namely, it was argued in Ref. [1] that in three spacetime dimensions the residual gauge freedom can be used to make the antisymmetric boundary condition for the field $A_{i}$ and the Hamiltonian contains a finite number of terms.

Making the final conclusion, we assert that the theory of lightcone QCD has the following features: 
- The boundary field $\gamma_{i}$ at $x^{-}=\infty$ plays the important role. It affects on physical scattering amplitudes beyond the leading order.

- The symplectic method is most useful in this case. The structure of the classical phase space is linear symplectic space.

- For 4D space-time and non-Abelian gauge group the Hamiltonian has infinite number of terms and infinite power over the coupling constant $g$.

- The quantum structure of lightcone QCD is still not clear. Beyond leader order the theory cannot be directly quantized. The careful analysis shows that a Poisson formulation has the problems with: canonical commutation relations, spatial invariance, and the boundary degrees of freedom in the Hamiltonian.

\section{Acknowledgments}

This work and the participation in QFTHEP'2011 was supported by RFFI 11-02-01395-a grant.

\section{References}

[1] A. V. Popov, Nucl. Phys. A 836 (2010) 136-158, arXiv:0908.0139.

[2] A. Kovner, M. Lublinsky, U. Wiedemann, JHEP 0706, 075 (2007), arXiv:0705.1713.

[3] W.-M. Zhang, A. Harindranath, Phys. Rev. D 48, 4868 (1993), arXiv:hep-th/9302119.

[4] W.-M. Zhang, A. Harindranath, Phys. Rev. D 48, 4881 (1993), arXiv:hep-th/9302107.

[5] P. Steinhardt, Ann. Phys. 128, 425 (1980).

[6] L. Faddeev, R. Jackiw, Phys. Rev. Lett. 60, 1692 (1988).

[7] V. O. Soloviev, J.Math.Phys. 34 (1993) 5747-5769, arXiv:hep-th/9305133.

[8] K. Bering, J.Math.Phys. 41 (2000) 7468-7500, arXiv:hep-th/9806249.

[9] V. O. Soloviev, arXiv:hep-th/9901112.

[10] A. V. Popov, arXiv:1107.4528. 